\author{
LESZEK ZYGNER \\ Państwowa Wyższa Szkoła Zawodowa w Ciechanowie \\ E-Mail: leszekzygner@gmail.com
}

\title{
DIE POLNISCHE HISTORISCHE MISSION IN GÖTTINGEN IN DEN JAHREN 2001-2009
}

Die Entstehung der Polnischen Historischen Mission am Max-Planck-Institut für Geschichte in Göttingen geht auf eine langjährige Zusammenarbeit der Thorner (Toruń) mit Göttinger Historikern zurück ${ }^{1}$. Eine besondere Rolle spielten in diesen Kontakten die seit 1981 in Thorn veranstalteten Tagungen „Ordines Militares. Colloquia Torunensia Historica“, die seit den 1980er Jahren immer mehr deutsche Historiker angezogen hatten, darunter auch einige aus dem Göttinger Wissenschaftsmilieu. Während der Tagungen in den 1970er und 1980er Jahren kamen zahlreiche deutsche Historiker nach Thorn, u. a. Hartmut Boockmann, Josef Fleckenstein, Otto Gerhard Oexle, Frank Rexroth, Lutz Fenske, Dieter Girgensohn und Peter Kriedte. Umgekehrt begaben sich die Thorner Historiker nach Göttingen. Seit der Mitte der 1980er Jahre wurde diese Stadt zum beliebten Bildungsziel zahlreicher Forscher von der Nikolaus-Kopernikus-Universität Thorn, denen seit 1983 auch Stipendien des Max-Planck-Instituts für Geschichte bewilligt wurden.

Der erste Thorner Stipendiat, der das Max-Planck-Institut für Geschichte auf Einladung des damaligen Institutsdirektors Rudolf Vierhaus sowie Peter Kriedtes besuchte, war Kazimierz Wajda (1983). In den darauffolgenden Jahren bewilligte man Stipendien an folgende Forscher: Zenon Hubert

${ }^{1}$ Mehr dazu L. Zygner, Die Zusammenarbeit von Historikern aus Thorn und Göttingen im vergangenen halben Jahrhundert, in: Bulletin der Polnischen Historischen Mission, 2 (2004), S. 285-295. 
Nowak (1987), Maksymilian Grzegorz (1988), Roman Czaja (1990), Janusz Tandecki (1991), Andrzej Radzimiński (1994), Wiesław Sieradzan (1995) und Piotr Oliński (1999). Zur selben Zeit begannen Stefan Kwiatkowski und Jarosław Wenta an der Georg-August-Universität Göttingen ihre wissenschaftlichen Forschungen unter Betreuung von Hartmut Boockmann. Es ist bemerkenswert, dass nicht nur Thorner, sondern auch Breslauer (Wrocław), Posener (Poznań), Warschauer (Warszawa) und Krakauer (Kraków) Historiker Göttingen aufsuchten. Trotzdem spielte Thorn in diesen Beziehungen von Anfang an die wichtigste Rolle. Die Intensivierung der Kontakte zwischen Göttingen und Thorn in den 1990er Jahren führte dazu, dass der Zusammenarbeit beider Historikergruppen ein mehr formaler Charakter verliehen wurde ${ }^{2}$.

Noch zu Lebzeiten Professor Hartmut Boockmanns, der das Seminar für Mittlere und Neuere Geschichte an der Universität Göttingen leitete, beschloss man, eine offizielle Zusammenarbeit zwischen dem von ihm geleiteten Institut und dem Institut für Geschichte und Archivistik der Universität Thorn aufzunehmen. Nach dem unerwarteten Tode Hartmut Boockmanns 1998 setzte sein Nachfolger Frank Rexroth das Konzept fort und unterzeichnete zusammen mit Janusz Tandecki von der Universität Thorn einen Vertrag, der den Austausch von Studenten und Forschern zwischen beiden Universitäten ermöglichte. Auf Anregung Otto Gerhard Oexles, des damaligen Direktors des Max-Planck-Instituts für Geschichte, sowie Zenon Hubert Nowaks von der Universität Thorn bemühte man sich um die Eröffnung der Polnischen Historischen Mission am Göttinger Institut, die nach dem Vorbild der schon bestehenden Einrichtungen wie der Mission Historique Française en Allemagne und des British Center for Historical Research in Germany funktionieren sollte. Auch nach dem plötzlichen Tod von Zenon Hubert Nowak 1999 wurde das Konzept fortgesetzt. Auf der polnischen Seite waren es zwei Thorner Historiker, Janusz Tandecki und Andrzej Radzimiński, die die Bemühungen Otto Gerhard Oexles um die Gründung der Mission unterstützten ${ }^{3}$. Mit ihrer Eröffnung und Orga-

2 Ibidem, S. 293-295; W. Sieradzan, Die wissenschaftliche Zusammenarbeit zwischen Professor Hartmut Boockmann und Professor Zenon Hubert Nowak, in: Bulletin der Polnischen Historischen Mission, 2 (2004), S. 305.

3 Hinzuzufügen ist, dass dem Direktor des Max-Planck-Instituts für Geschichte, Otto Gerhard Oexle, in Anerkennung seiner herausragenden Verdienste 2002 die Ehrendoktorwürde (Doctor honoris causa) der Nikolaus-Kopernikus-Universität Thorn verliehen 
nisation setzte sich Leszek Zygner von der Universität Thorn auseinander, der von August 2001 bis Januar 2009 Leiter der Mission war.

Nach den ersten Tätigkeitsmonaten der neuen Institution organisierte man am 21. Februar 2002 eine offizielle Eröffnungsfeier in Anwesenheit von Vertretern der Staats- und Landesbehörden sowie der Konsulate und zahlreicher Gäste aus Deutschland und Polen ${ }^{4}$. Die Tätigkeit der Mission beaufsichtigte anfänglich der Wissenschaftliche Beirat des Max-Planck-Instituts für Geschichte, danach der Wissenschaftliche Beirat der Polnischen Historischen Mission, der sich erstmals am 23. August 2005 versammelte 5 Zum Beirat gehörten: Janusz Tandecki (Thorn) als Vorsitzender, Andrzej Radzimiński (Thorn), Wojciech Fałkowski (Warschau), Tomasz Jasiński (Posen), Jan Rydel (Krakau), Otto Gerhard Oexle (Göttingen) und Helmut Flachenecker (Würzburg).

Im Zusammenhang mit der Entstehung und der Tätigkeit der Polnischen Historischen Mission in Göttingen wurde auch ihre Finanzierung diskutiert. Es ging vor allem um die Gewinnung von Mitteln für Forschungsprojekte, wissenschaftliche Tagungen, Veröffentlichungen, wissenschaftliche Stipendien für die polnischen Historiker, die nach Göttingen kommen wollten, sowie für das Gehalt des Missionsleiters und seine Büroausgaben. Dank der Bemühungen des Direktors des Max-Planck-Instituts für Geschichte, Otto Gerhard Oexle, wurde die Polnische Historische Mission in den ersten drei Jahren vor allem aus den Mitteln der Gerda Henkel Stiftung aus Düsseldorf sowie der Max-Planck-Gesellschaft mitfinanziert. Da Polen zu diesem Zeitpunkt noch kein Mitgliedstaat der Europäischen Union war, wurde der Leiter der Mission vom 1. August 2001 bis zum 31. Juli 2004 offiziell als wissenschaftlicher Mitarbeiter des Max-Planck-Instituts für Geschichte in Göttingen angestellt und erhielt ein Forschungsstipendium der Gerda Henkel Stiftung. Aus den Mitteln des Max-Planck-Instituts für Geschichte wurden dagegen die Stipendien für die polnischen Historiker, die nach Göttingen kamen, sowie einige der damals organisierten Tagungen finanziert. Die Bü-

wurde. Mehr dazu siehe: Homines et Historia. Doktorat honoris causa dla prof. dr hab. Otto Gerharda Oexle dyrektora Max-Planck-Institut für Geschichte w Getyndze, (2003).

${ }^{4}$ L. Zygner, Die Polnische Historische Mission in Göttingen - eine neue polnische Institution der Geschichtswissenschaft in Deutschland, in: Inter Finitimos. Jahrbuch zur Deutsch-Polnischen Beziehungsgeschichte, 1 (2003), S. 152-153.

${ }^{5}$ Chronik der Polnischen Historischen Mission, in: Bulletin der Polnischen Historischen Mission, 4 (2007), S. 157. 
roausgaben der Mission sowie die Veröffentlichungskosten des Bulletins der Polnischen Historischen Mission übernahm die Nikolaus-Kopernikus-Universität Thorn. Die meisten wissenschaftlichen Publikationen, die in dieser Zeitspanne erschienen, wurden aus den Mitteln des Max-Planck-Instituts für Geschichte finanziert. An den Kosten der übrigen Tagungen, Seminare und Veröffentlichungen beteiligten sich auch die Universität Göttingen, die Deutsch-Polnische Gesellschaft Göttingen e.V., die Stiftung für polnische Wissenschaft, die Universität Breslau (Wrocław), das Generalkonsulat der Republik Polen in Hamburg und das Generalkonsulat der Bundesrepublik Deutschland in Breslau. Seit 2004 wurde die Polnische Historische Mission in den drei darauffolgenden Jahren vom polnischen Wissenschafts- und Informatisierungsministerium, dem Max-Planck-Institut für Geschichte und der Universität Thorn finanziell unterstützt. Die zwei letztgenannten Institutionen übernahmen auch die Veröffentlichungskosten der Reihe Prussia Sacra. Seit 2007 wurden die Stipendien für die polnischen Forscher aus den Mitteln verschiedener Stiftungen und den Eigenmitteln der Gäste, die nach Göttingen kamen, finanziert, und die Unterstützung der Mission selbst übernahm die Universität Thorn. An einigen Veranstaltungen, wie etwa Tagungen, Seminaren und Vorlesungen, beteiligten sich teilweise auch das Seminar für Mittlere und Neuere Geschichte der Universität Göttingen und die Deutsch-Polnische Gesellschaft Göttingen e.V.

Über die dynamische Entwicklung der Mission in den ersten sechs Jahren ihres Bestehens entschieden nicht nur die finanzielle Unterstützung, sondern auch das Engagement und die Zusammenarbeit mehrerer Persönlichkeiten. Neben dem Leiter und den Mitgliedern des Wissenschaftlichen Beirats wurde die Polnische Historische Mission von Anfang an von den Direktoren des Max-Planck-Instituts für Geschichte, Otto Gerhard Oexle und Hartmut Lehmann, sowie von den wissenschaftlichen Mitarbeitern des Instituts unterstützt, welche die polnischen Forscher, also die zukünftigen Stipendiaten, nach Göttingen einluden: Caspar Ehlers, Helmut Flachenecker, Bernhard Jussen, Peter Kriedte, Nathalie Kruppa, Alf Lüdtke, Hans Medick, Jürgen Schlumbohm, Peter Schumann, Martial Staub, Thomas Szabó. Neben ihnen beteiligten sich an der Tätigkeit der Mission auch die Professoren der Universität in Göttingen: Frank Rexroth, Hedwig Röckelein und Manfred Hildermeier, ferner die Göttinger emeritierten Wissenschaftler Rudolf Vierhaus, Irene Crusius, Rudolf Joachim von Thadden sowie die Mitglieder der Deutsch-Polnischen Gesellschaft Göttingen e. V. mit den 
Vorstandsvorsitzenden dieser Gesellschaft, Reinhard Caspari und Dieter Wiedemann, an der Spitze. Nach der Abschaffung des Max-Planck-Instituts für Geschichte und der Gründung eines neuen Max-Planck-Instituts zur Erforschung multireligiöser und multiethnischer Gesellschaften in Göttingen im Jahre 2007 setzte die Polnische Historische Mission ihre Tätigkeit weiterhin fort, doch in einem begrenzten Umfang. Offiziell wurde das Büro der Mission im Januar 2009 geschlossen. In der Mitte des Jahres 2009 erschien die letzte (fünfte) Nummer des Bulletins der Polnischen Historischen Mission in Göttingen. Nach einer mehrmonatigen Pause, im September 2009, wurde die Polnische Historische Mission aufs Neue eröffnet, und zwar an der Julius-Maximilians-Universität in Würzburg.

In den Jahren 2002-2007 bewilligte man im Rahmen der Tätigkeit der Polnischen Historischen Mission über 70 ein- und zweimonatige Stipendien an Forscher folgender historischer Institute Polens: der Nikolaus-Kopernikus-Universität Thorn, der Universität Breslau, der Universität Warschau, der Universität Stettin (Szczecin), der Jagiellonen-Universität Krakau, der Adam-Mickiewicz-Universität Posen, der Katholischen Universität Lublin, der Maria-Curie-Skłodowska-Universität Lublin, der Universität Białystok, der Universität Oppeln (Opole), der Schlesischen Universität Kattowitz (Katowice), der Pommerschen Akademie Stolp (Słupsk), der Świętokrzyski-Akademie Kielce, der Medizinischen Akademie Danzig (Gdańsk), des Instituts für Geschichte der Polnischen Akademie der Wissenschaften, des Instituts für Archäologie und Ethnologie der Polnischen Akademie der Wissenschaften. Bis 2008 erfolgten zudem 13 Stipendiatenaufenthalte in der Mission. Die Stipendiaten wurden von der Alexander von Humboldt-Stiftung, der Robert Bosch Stiftung sowie von der Nikolaus-Kopernikus-Universität Thorn, der Jagiellonen-Universität Krakau und der Universität Ermland-Masuren Allenstein (Olsztyn), ferner aus den Mitteln des Instituts für Archäologie und Ethnologie der Polnischen Akademie der Wissenschaften sowie aus Eigenmitteln finanziell unterstützt ${ }^{6}$. Neben der Beaufsichtigung von Forschungsprojekten der Stipendiaten realisierte der

${ }^{6}$ Die polnischen Gäste und Stipendiaten des Max-Planck-Instituts für Geschichte 2002, in: Bulletin der Polnischen Historischen Mission, 1 (2002), S. 92-93; Die polnischen Gäste und Stipendiaten des Max-Planck-Instituts für Geschichte 2003, in: Bulletin der Polnischen Historischen Mission, 2 (2004), S. 155-156; Die polnischen Gäste und Stipendiaten des Max-Planck-Instituts für Geschichte 2004, in: Bulletin der Polnischen Historischen Mission, 3 (2005), S. 106-108; Die polnischen Gäste und Stipendiaten des Max-Planck-Instituts für 
Leiter der Mission sein eigenes wissenschaftliches Projekt über die Kirchenreform in Mittelosteuropa im Spätmittelalter, finanziert aus den Mitteln der Gerda Henkel Stiftung.

Für die wissenschaftliche Tätigkeit der Mission, die erweiterten Kontakte zu verschiedenen historischen Einrichtungen in Deutschland und in anderen Ländern spielten die von der Mission veranstalteten wissenschaftlichen Tagungen und die danach herausgegebenen Tagungsbände eine besondere Rolle. Schon 2002, ein Jahr nach der Eröffnung der Mission, organisierte man zusammen mit dem Max-Planck-Institut für Geschichte, der Mission Historique Française en Allemagne und dem British Center for Historical Research in Germany ein internationales Kolloquium junger Mediävisten unter dem Titel Standorte und künftige Entwicklungen der europäischen Mediävistik. Perspektiven der jungen Generation, an dem Historiker aus Deutschland, Frankreich, Polen, Großbritannien und Russland teilnahmen. 2003 fand ein internationales Kolloquium Die Deutung der mittelalterlichen Gesellschaft in der Moderne (19.-20. Jahrhundert) statt, an dem sich Historiker aus Deutschland, Frankreich, Großbritannien und Polen beteiligten, sowie das Kolloquium Bilder gedeuteter Geschichte. Das Mittelalter in der Kunst und Architektur der Moderne, veranstaltet von der Polnischen Historischen Mission und dem Lehrstuhl für Literatur und Kultur Mitteleuropas an der Universität Miskolc (Ungarn). Unter Mitwirkung des Generalkonsulats der Republik Polen in Hamburg sowie dem Generalkonsulat der Bundesrepublik Deutschland in Breslau fand 2003 die Tagung Niedersachsen - Niederschlesien. Der Weg beider in die Geschichte statt, veranstaltet von der Polnischen Historischen Mission, dem Max-Planck-Institut für Geschichte und der Universität Breslau. In Zusammenarbeit mit dem Forschungsprojekt Germania Sacra, realisiert vom Max-Planck-Institut für Geschichte, organisierte man zwei internationale wissenschaftliche Tagungen zur Kirchengeschichte: Partikularsynoden im Spätmittelalter.

Geschichte 2005, in: Bulletin der Polnischen Historischen Mission, 4 (2007), S. 159-160; Die polnischen Gäste und Stipendiaten des Max-Planck-Instituts für Geschichte und der Polnischen Historischen Mission 2006-2008, in: Bulletin der Polnischen Historischen Mission, 5 (2009), S. $175-177$.

${ }^{7}$ Siehe: Bilder gedeuteter Geschichte. Das Mittelalter in der Kunst und Architektur der Moderne, hg. v. O. G. Oexle et al., 1-2 (2004); Niedersachsen und Niederschlesien. Der Weg beider in die Geschichte, hg. v. W. Mrozowicz, L. Zygner, (2005); Die Deutung der mittelalterlichen Gesellschaft in der Moderne, hg. v. N. Fryde et al., (2006). 
Deutschland, Polen und Tschechien im Vergleich (2004) und Pfarreien in Mitteleuropa im Mittelalter. Deutschland, Polen, Tschechien und Ungarn im Vergleich (2006) ${ }^{8} .2007$ wurden unter Mitwirkung des Seminars für Mittlere und Neuere Geschichte der Georg-August-Universität Göttingen sowie der Mission Historique Française en Allemagne eine internationale Doktorandenwerkstatt unter dem Titel Übersetzen als geschichtswissenschaftliches Problem sowie eine internationale Forschungswerkstatt Mittelalterliche Eliten und Kulturtransfer östlich der Elbe vorbereitet. In Zusammenarbeit mit dem Arbeitskreis für Agrargeschichte wurde schließlich am Sitz der Mission eine Tagung unter dem Titel Tiernutzung in der Landwirtschaft vom Mittelalter bis zur Moderne (2007) veranstaltet ${ }^{9}$.

Neben den Tagungen in den Jahren 2001-2008 organisierte die Polnische Historische Mission in Zusammenarbeit mit dem Seminar für Mittlere und Neuere Geschichte der Georg-August-Universität und der Deutsch-Polnischen Gesellschaft Göttingen e.V. in Göttingen 40 Vorlesungen polnischer Historiker, die die folgenden Institutionen repräsentierten: die Nikolaus-Kopernikus-Universität Thorn, die Universität Warschau, die Jagiellonen-Universität Krakau, die Universität Breslau, die Adam-Mickiewicz-Universität Posen, die Universität Stettin, die Universität Oppeln, die Pädagogische Akademie Krakau, die Pommersche Akademie Stolp, das Institut für Geschichte der Polnischen Akademie der Wissenschaften Warschau und Posen, das Wissenschaftliche Zentrum der Polnischen Akademie der Wissenschaften Berlin sowie die Universitäten Hamburg und Bonn. Die wissenschaftlichen Mitarbeiter des Max-Planck-Instituts für Geschichte hielten sieben Vorträge an den polnischen Universitäten Thorn, Warschau und Breslau ${ }^{10}$.

Ein Meilenstein in der Geschichte der Polnischen Historischen Mission in Göttingen, und zwar neben den Tagungsbänden, die in Zusammenarbeit mit dem Max-Planck-Institut für Geschichte herausgegeben wurden, ist

8 Siehe: Partikularsynoden im späten Mittelalter, hg. v. N. Kruppa, L. Zygner, (2006); Pfarreien im Mittelalter. Deutschland, Polen, Tschechien und Ungarn im Vergleich, hg. v. N. Kruppa unter Mitwirkung v. L. Zygner, (2008).

9 Chronik der PHM, in: Bulletin der Polnischen Historischen Mission, 1 (2002), S. 91; ibidem, 2 (2004), S. 151-155; ibidem, 3 (2005), S. 104-106; ibidem, 5 (2009), S. 172-174.

${ }^{10}$ Chronik der PHM, in: Bulletin der Polnischen Historischen Mission, 1 (2002), S. 91-92; ibidem, 2 (2004), S. 151-155; ibidem, 3 (2005), S. 103-106; ibidem, 4 (2007), S. 157-158; ibidem, 5 (2009), S. 171-174. 
die bis heute bestehende Reihe Prussia Sacra. Historische Beschreibung der Kirche im Deutschordensland Preußen, die auf Anregung des damaligen wissenschaftlichen Leiters des Projektes Germania Sacra, Helmut Flachenecker, und Andrzej Radzimiński, des Leiters des Lehrstuhls für Kirchengeschichte am Institut für Geschichte und Archivistik der Universität Thorn, entstand. In der Göttinger Zeit der Polnischen Historischen Mission erschienen drei Bände dieser Reihe, mitfinanziert vom Max-Planck-Institut für Geschichte und der Nikolaus-Kopernikus-Universität Thorn ${ }^{11}$. Aus den Mitteln des Instituts für Geschichte und Archivistik der Universität Thorn wurden fünf Nummern des zweisprachigen Bulletins der Polnischen Historischen Mission finanziert, herausgegeben von Peter Schumann (Max-Planck-Institut für Geschichte), Leszek Zygner (Polnische Historische Mission) sowie Włodzimierz Zientara (Nikolaus-Kopernikus-Universität Thorn). Weitere Nummern dieser Zeitschrift werden von der Polnischen Historischen Mission in Würzburg auf Deutsch bzw. Englisch herausgegeben und die einzelnen Artikel mit Zusammenfassungen auf Polnisch, Deutsch und Englisch publiziert.

Übersetzt von

Liliana Lewandowska

\author{
POLSKA MISJA HISTORYCZNA \\ W GETYNDZE W LATACH 2001-2009
}

STRESZCZENIE

Polska Misja Historyczna działająca w latach 2001-2009 przy Instytucie Historycznym Maxa Plancka w Getyndze powstała na podstawie trwającej od wielu lat współpracy historyków toruńskich i getyńskich. W czasie jej działalności przydzielano stypendia dla polskich badaczy, organizowano międzynarodowe konferencje naukowe i warsztaty dla doktorantów oraz studentów, a także odczyty polskich historyków. W okresie działalności Misji w Getyndze ukazało się również pięć numerów Biuletynu Polskiej Misji Historycznej, a także trzy tomy serii Prussia Sacra. Historische Beschreibung der Kirche im Deutschordensland Preußen.

11 Siehe: M. Glauert, Das Domkapitel von Pomesanien (1284-1527), (2003); R. Biskup, Das Domkapitel von Samland (1285-1525), (2007); M. Jarzebowski, Die Residenzen der preußischen Bischöfe bis 1525, (2007). 


\title{
DiE POLNISCHE HISTORISCHE MISSION IN GÖTTINGEN \\ IN DEN JAHREN 2001-2009 \\ ZUSAMMENFASSUNG
}

Die Polnische Historische Mission, die in den Jahren 2001-2009 am Max-Planck-Institut für Geschichte in Göttingen angesiedelt war, wurde aufgrund der langjährigen Zusammenarbeit von Historikern aus Toruń und Göttingen gegründet. Während ihrer Tätigkeit wurden Stipendien für polnische Forscher vergeben, internationale wissenschaftliche Tagungen und Workshops für Doktoranden und Studenten veranstaltet sowie Vorträge von polnischen Historikern gehalten. Es erschienen auch fünf Bände des Bulletins der Polnischen Historischen Mission sowie drei der Reihe Prussia Sacra. Historische Beschreibung der Kirche im Deutschordensland Preußen.

\author{
Übersetzt von \\ Renata Skowrońska
}

\section{POLISH HISTORICAL MISSION IN GÖTTINGEN IN THE YEARS 2001-2009 \\ SUMMARY}

The Polish Historical Mission operating in the years 2001-2009 at the Max Planck Institute for History in Göttingen was set up on the basis of the long-lasting cooperation between the historians of Torun and Göttingen. During its activity scholarships were granted to Polish scholars, international scientific conferences and workshops for $\mathrm{PhD}$ students and students along with lectures given by Polish historians were organized. During this period five editions of the Bulletin of the Polish Historical Mission and three volumes of the series Prussia Sacara were published.

\author{
Translated by \\ Agnieszka Chabros
}

\section{SŁOWA KLUCZOWE / SCHLAGWORTE / KeYWORdS}

- Polska Misja Historyczna; Getynga; współpraca historyków polskich i niemieckich

- Polnische Historische Mission; Göttingen; Zusammenarbeit polnischer und deutscher Historiker

- The Polish Historical Mission; Göttingen; cooperation of Polish and German historians 


\section{BIBLIOGRAFIA / BIBLIOGRAFIE / BIBLIOGRAPHY}

\section{LITERATURA / LITERATUR / LITERATURE}

Bilder gedeuteter Geschichte. Das Mittelalter in der Kunst und Architektur der Moderne, hg. v. O. G. Oexle et al., 1-2 (2004).

Biskup R., Das Domkapitel von Samland (1285-1525), (2007).

Chronik der PHM, in: Bulletin der Polnischen Historischen Mission, 1 (2002), S. 90-92 und 2 (2004), S. 151-155 und 3 (2005), S. 103-106, 4 (2007), S. 157-158 und 5 (2009), S. 171-174.

Die Deutung der mittelalterlichen Gesellschaft in der Moderne, hg. v. N. Fryde et al., (2006).

Die polnischen Gäste und Stipendiaten des Max-Planck-Instituts für Geschichte, in: Bulletin der Polnischen Historischen Mission, 1 (2002), S. $92-93$ und 2 (2004), S. 155-156 und 3 (2005), S. 106-108 und 4 (2007), S. 159-160 und 5 (2009), S. 175-177.

Glauert M., Das Domkapitel von Pomesanien (1284-1527), (2003).

Homines et Historia. Doktorat honoris causa dla prof. dr hab. Otto Gerharda Oexle dyrektora Max-Planck-Institut für Geschichte w Getyndze, (2003).

Jarzebowski M., Die Residenzen der preußischen Bischöfe bis 1525, (2007).

Niedersachsen und Niederschlesien. Der Weg beider in die Geschichte, hg. v. W. Mrozowicz, L. Zygner, (2005).

Partikularsynoden im späten Mittelalter, hg. v. N. Kruppa, L. Zygner, (2006).

Pfarreien im Mittelalter. Deutschland, Polen, Tschechien und Ungarn im Vergleich, hg. v. N. Kruppa unter Mitwirkung v. L. Zygner, (2008).

Sieradzan W., Die wissenschaftliche Zusammenarbeit zwischen Professor Hartmut Boockmann und Professor Zenon Hubert Nowak, in: Bulletin der Polnischen Historischen Mission, 2 (2004), S. 305.

Zygner L., Die Polnische Historische Mission in Göttingen - eine neue polnische Institution der Geschichtswissenschaft in Deutschland, in: Inter Finitimos. Jahrbuch zur Deutsch-Polnischen Beziehungsgeschichte, 1 (2003), S. 152-153.

Zygner L., Die Zusammenarbeit von Historikern aus Thorn und Göttingen im vergangenen halben Jahrhundert, in: Bulletin der Polnischen Historischen Mission, 2 (2004), S. 285-295. 